\title{
ANALYSIS OF PACKETS DELAY IN WIRELESS DATA NETWORKS
}

\author{
Aleksandr Krivchenkov ${ }^{1}$, Dmitry Sedykh ${ }^{1,2}$ \\ ${ }^{1}$ Transportation and Telecommunication Institute \\ Lomonosova str. 1, LV-1019, Riga, Latvia \\ Ph.: +371 29210689, e-mail: krivcenkovs.a@tsi.lv \\ ${ }^{2}$ Karolin SIA, Riga, Latvia \\ Ph.: +371 29473411,e-mail: dmitrijs.sedihs@inbox.lv
}

\begin{abstract}
The networks with wireless links for automation control applications traffic transmission when packets have small size and application payload is predictable are under consideration. Analytical model for packets delay on their propagation path through the network is proposed. Estimations for network architectures based on WiFi and Bluetooth wireless technologies are made. The specifications for physical layer $802.11 \mathrm{a} / \mathrm{b} / \mathrm{g} / \mathrm{n}$ and 802.15 .1 are under consideration. Analytical and experimental results for delivered network bandwidth for different network architecture, traffic structure and wireless technologies were compared to validate that basic mechanisms are correctly taken into account in the model. It is shown that basic effects are taken into account and further accuracy "improvement" of the model will give not more than $5 \%$. As a result that is important for automation control applications we have reliably received the lowest possible level for packets delay in one wireless link. For 802.11 it is of order of $0.2 \mathrm{~ms}$, for 802.15 .1 it is $1.25 \mathrm{~ms}$ and is true when application packet can be transferred by one data frame.
\end{abstract}

Keywords: WLAN, WiFi, Bluetooth, 802.11, 802.15.1, packet delay

\section{Introduction}

Wireless Local Area Networks based on 802.11technology (WiFi) and 802.15.1 (Bluetooth) have become quite popular and widespread. The nature of links based on the radio channel and the access to the shared resource of this channel cause variable available bandwidth, variable packet delay and loss rate. If changes in these parameters are not taken into account it may prevent to the correct operation of the networked time-sensitive applications, such as multimedia or control applications.

In the automation area, there is a clear trend promoting the use of wireless control channels in the factory floor. Automation control applications bring forward the demands to wireless links structure. It is obviously that delay in the delivery of packets introduced by the network links may degrade control applications performance or just makes such control quite impossible. Therefore, a good estimation of the network latency together with network bandwidth will facilitate robust system designs.

In this paper the analytical model for the estimation of possible delay of packets in links and provided network bandwidth with "acceptable" performances for several WiFi technologies $(802.11 \mathrm{a} / \mathrm{b} / \mathrm{g} / \mathrm{h})$ and Bluetooth technology $(802.15 .1)$ is considered. The approach in the model follows the one in (Krivchenkov and Saltanovs, 2014) but essentially includes the calculations for technology 802.15.1. The differences between the contention mechanism (competition) for radio recourse in the links for 802.11 and resource reservation mechanism for 802.15.1 are taken into account.

The experimental data for different Access points (AP) and wireless host's adapters were collected in set of experiments. Analytical and practical results for delivered network bandwidth for different network architecture, traffic structure and wireless technologies were compared to validate that basic mechanisms are correctly taken into account in the model, and it may be recommended as a useful tool for network designers.

\section{Model of data transfer network}

\subsection{Common characteristics of the model}

In our model we are taken into account the network architecture and traffic structure, we consider that there are the streams of packets with data between communicating nodes: 


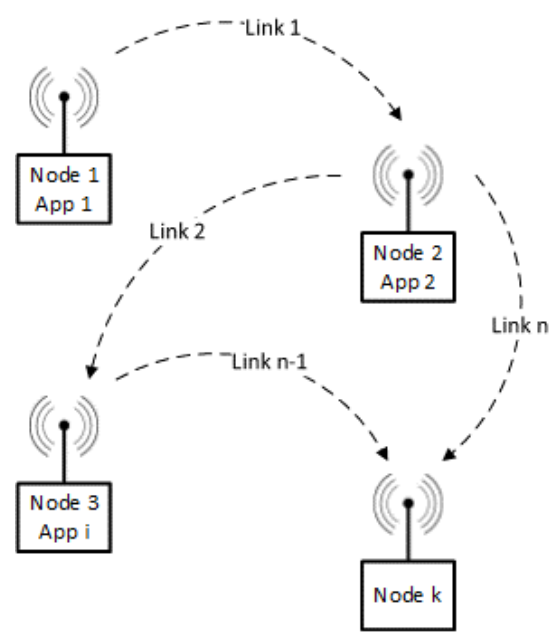

Figure 1. The architecture of network and structure of traffic

The sources of packets (see Figure 1) are Applications working in the nodes. There are links between nodes and paths of packets between different nodes. For the general definition we introduce the next entities: $\hat{R}_{\text {App }}$ - the traffic structure (or payload) on the level of applications; $\hat{S}$-the architecture of network, is defined by applications demands and wireless technologies possibilities; $\hat{P}$-wireless technologies parameters; $\hat{I}$ - parameters of noise in wireless channels; $T_{\text {Frame }}(\hat{P}, l)$ - frame (packet on Data Link layer) transfer time through a link, where $l$ - packet size on application layer; $\operatorname{PER}(\hat{I}, \hat{P}, l)$-probability of error in a packet when it is transmitted through a link; $D_{\text {App }}\left(\hat{S}, \hat{R}_{\text {App }}, T_{\text {Frame }}\right)=D_{\text {App }}\left(\hat{S}, \hat{R}{ }_{A p p}, \hat{P}, \hat{I}, l\right)-$ the application packets delay on a path.

In these notations the task of "effective" network design for real time applications formally will be as follows. For the given range of $\hat{R}_{A p p}, l$ and $\hat{I}$ the network with $\hat{S}$ and $\hat{P}$ to be provided in what for the delays of packets it is guaranteed that $\hat{D}_{A p p}\left(\hat{S}, \hat{R}_{A p p}, \hat{P}, \hat{I}, l\right) \leq \hat{D}_{0}$ or the delays will be not greater than some given delays.

\subsection{Analytical model for estimations}

\section{Payload characteristics}

Payload in the model is characterized by several obvious parameters:

$R_{A p p P}$ - number of packets generated by application per unit time (packet per second); $l$ - applications packet size (byte). So, the bit rate generated on application level will be:

$$
R_{\text {App }}=R_{\text {AppP }} \cdot 8 l
$$

It is measured in bits per second (bps) or Kbps, Mbps.

The next "enhancement of payload" on data transfer channel is defined by protocols used on Transport and Network layers. For every network technology Data Link layer also adds some bytes. For: $\Delta l_{\text {Transport }}$ - additional bytes of transport protocol; $\Delta l_{\text {Network }}$ - additional bytes of network protocol; $\Delta l_{\text {Frame }}$ - additional bytes of data link protocol. The bit rate on Physical level will be:

$$
R_{\text {App }}^{\text {Phy }}=R_{\text {AppP }} \cdot 8\left(l+\Delta l_{\text {Transport }}+\Delta l_{\text {Network }}+\Delta l_{\text {Frame }}\right)
$$


Late we will take into account later that for UDP protocol $\Delta l_{\text {Transport }}=8$, for IP protocol $\Delta l_{\text {Network }}=20$ and for an example for 802.11 frames $\Delta l_{\text {Frame }}=36$.

To characterize the process of packets transfer we introduce two more parameters:

$T_{\text {Frame }}$ - the time of frame transfer through the link and $R_{\text {Frame }}$ - the number of frames transmitted per unit time. It is important that if one application packet is transferred exactly by one frame (for an example wellknown fragmentation mechanism for transmission technology is not used) we have:

$$
R_{\text {App }}=R_{\text {AppP }} \cdot 8 \mathrm{l}=R_{\text {Frame }} \cdot 8 \mathrm{l}
$$

We define also dimensionless parameter that characterizes "busyness" of channel. Often it is also called "payload" or "utilization":

$\rho=R_{\text {Frame }} T_{\text {Frame }}$

This quantity is in the range $0 \leq \rho \leq 1$. When $\rho=0$ no frames are transmitted, when $\rho=1$ the channel is busy in every moment of time (100\% utilized).

\section{Packet delay}

For packets transfer process some model of service can be introduced. In such model application packets are just requests for service and the intensities of requests are $\lambda_{i}$ (index $i$ enumerates applications). Service is provided in links and intensities of services are $\mu_{\text {ni }}$ (index n enumerates links). So, the set of links on the path give us a set of service nodes:

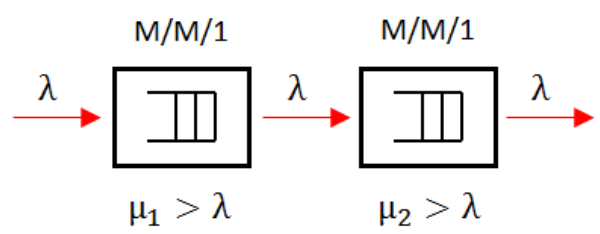

Figure 2. Service model for the path of 2 links

According to the Queueing Theory, when requests with intensity $\lambda$ are coming on sequence of serving nodes where service is made with intensity $\mu$ and when time intervals between requests and time of request's service are exponentially distributed (so called $\mathrm{M} / \mathrm{M} / 1$ model) the average service time in one node will be:

$\bar{t}=\frac{1}{\mu}+\frac{1}{\mu} \cdot \frac{\rho}{1-\rho}$, where $\rho=\lambda / \mu$ and $0 \leq \rho<1$.

Simplification of real world processes in this model for packets delay is obvious, but it is accepted in many cases for the estimations in computer networks design. This model can be "easy improved". For an example we may use $\mathrm{M} / \mathrm{G} / 1$ instead $\mathrm{M} / \mathrm{M} / 1$ approximation, when service tame has an arbitrary distribution and the average service time instead (5) will be:

$\bar{t}=\frac{1}{\mu}+\frac{1}{\mu} \cdot \frac{\rho\left(1+c^{2}\right)}{2(1-\rho)}$, where $c=\frac{\sqrt{D\left(t_{s}\right)}}{\bar{t}_{s}}$

$D\left(t_{s}\right)$ - the variance of service time (for exponential distribution of service time $\mathrm{c}=1$ ).

Now let us define what "payload" or "utilization" will be in (5) and (6). If some link has its own serving recourse and it not shares this recourse with other links due to definition (4) utilization of link will be:

$\rho_{n}=\sum_{\{i\}_{n}} R_{\text {Frame }_{n i}} T_{\text {Frame }_{n i}}$ 
$\{i\}_{n}$-means that summing to be done taking into account those applications witch packets will be transferred through link $\mathrm{n}$. If serving recourse is shared with other links in (7) will be additional term:

$\rho_{n}=\sum_{\{i\}_{n}} R_{\text {Frame }_{n i}} T_{\text {Frame }_{n i}}+\sum_{\{i\}_{n}^{\prime},\left\{n^{\prime}\right\}_{n}} R_{\text {Frame }_{n^{\prime} i}} T_{\text {Frame }_{n^{\prime} i}}$

$\left\{n^{\prime}\right\}_{n}$ - denotes summing for all those links witch use the same serving recourse as link $\mathrm{n}$ has.

For packets delay on some path $\{n\}_{i}$ using (6) and (8) we have:

$D_{A p p_{i}}=\sum_{\{n\}_{i}} D_{A p p_{n i}}=\sum_{\{n\}_{i}^{i}} T_{\text {Frameni }_{n i}}\left(1+\frac{\rho_{n}\left(1+c^{2}\right)}{2\left(1-\rho_{n}\right)}\right)$

In our model (9) gives the relationship between packets delays $D_{A p p}$ and network and traffic structure. There is some average time for frames transfer $T_{\text {Frame }}$. And payloads that applications generate $R_{\text {App }}$ denote payloads calculated for frames per unit time $R_{\text {Frame }}$. Relationship between $R_{\text {App }}, R_{\text {AppP }}, R_{\text {Frame }}$ are given in (1),(3).

For the simplification of understanding (9) let us consider the examples.

Example 1. Node1 is connected through WiFi channel to AP, transmits to Node2. Node2 is connected to AP with wired network. If we neglect with the delay in wired network, (9) and c=1(exponential distribution of $T_{\text {Frame }}$ ) gives:

$D_{\text {App }}=T_{\text {Frame }} \cdot \frac{1}{1-R_{\text {AppP }} \cdot T_{\text {Frame }}}$

Example 2. Node1 and Node2 are in the same BSS (Basic Service Set for 802.11) and Node2 transmits to Node1:

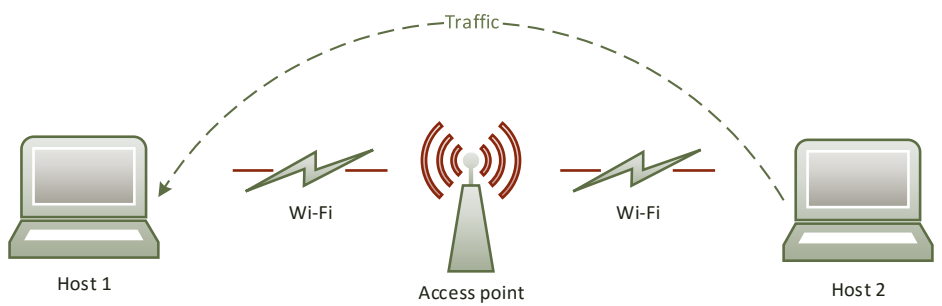

Figure 3. Structures of network and traffic for Example 2

We have 2 wireless links for this case and (9) gives:

$$
D_{\text {App }}=2 \cdot T_{\text {Frame }} \cdot \frac{1+\left(c^{2}-1\right) \cdot R_{\text {AppP }} \cdot T_{\text {Frame }}}{1-2 \cdot R_{\text {AppP }} \cdot T_{\text {Frame }}}
$$

Example 3. Node1 and Node2 are in the same BSS. Node 1 transmits to Node2 and Node2 transmits to Node1. If characteristics of application traffic for Node1 and Node2 are the same, the delay of packets in both directions from (9) will be:

$$
D_{\text {App }}=2 \cdot T_{\text {Frame }} \cdot \frac{1+2 \cdot\left(c^{2}-1\right) \cdot R_{\text {AppP }} \cdot T_{\text {Frame }}}{1-4 \cdot R_{\text {AppP }} \cdot T_{\text {Frame }}}
$$

On Figure 4 we have presented calculated values for different examples (Ex2, Ex3) and models (M/M/1, M/G/1, and simulation model "802.11g model Ex2"). 


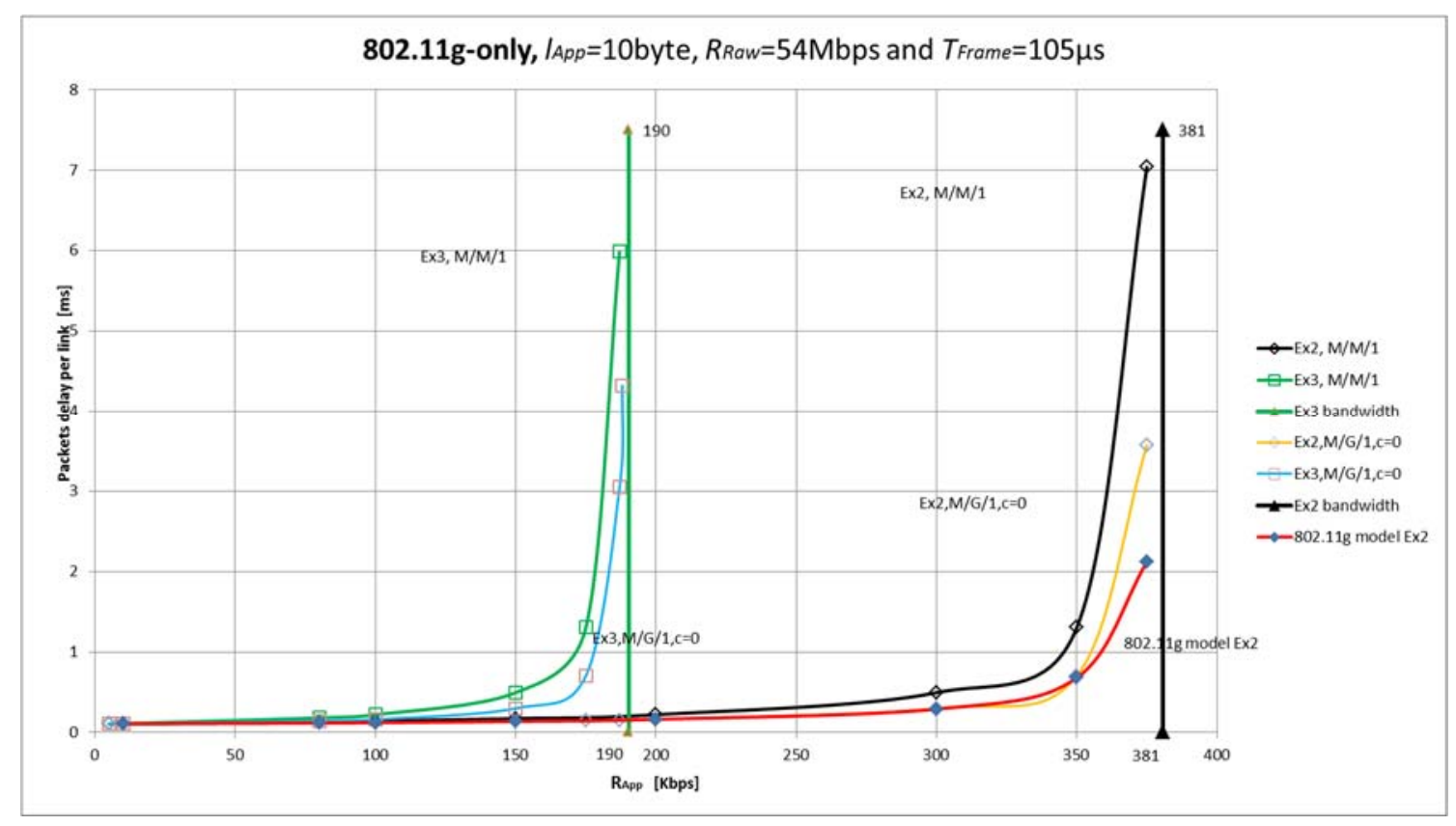

Figure 4. Calculated values for packets delays and different architectures

Thus, for different architectures there are common features. There is a limit of bit rate for applications and near this limit the delays of packets are sharply enhanced; so wireless channel has a bandwidth; minimal possible delay is defined by average frame time $T_{\text {Frame }}$. Bandwidth of channel is definitely related with "payload" (or utilization) given for the model in equation (8). Models give the significantly different values when utilization grater then 0.9 . So, simplest model $(M / M / 1)$ is applicable for the estimations in the range for $\rho \sim 0-0.9$. For the practice (packet delay needs to be of order $T_{\text {Frame }}$ ) the range $\rho \sim 0 \div 0.5$ is recommended and "practical" bandwidth may be calculated when $\rho=0.5$.

\subsection{Frame transfer time}

In previous pats of this paper we have discussed a simple model for the packets delay (and hence maximum throughput) for wireless networks. It was emphasized that for successful estimations we need to have reliable knowledge about $T_{\text {Frame }}$ for the technology that will be used in the network. In this section we discuss this parameter.

\section{Frame transfer time for 802.11}

In recent paper (Krivchenkov and Saltanovs, 2014) it was shown that for 802.11 networks a comparison of frame transfer time can move beyond a simple comparison of nominal bit rates $R_{\text {raw }}$ for different PHY (physical layer) specifications $802.11 \mathrm{a} / \mathrm{b} / \mathrm{g} / \mathrm{n}$. Following to the to 802.11 specifications (Gast, 2002), clarifying article (Gast, 2003) and publication (Qiang Ni, 2005) we try to take into account the transmission of small UDP packets and differences for MAC layer. We tried to consider the frame transfer mechanism. It was shown the importance of concurrent principle for the access to the radio channel and transactional nature of frame transfer. For the 802.11 $T_{\text {Frame }}$ estimations are summarized in next table.

Table 1. Relationships for $T_{\text {Frame }}$ estimations for different 802.11 specifications. UDP packets, application packet size $l$ [bytes], Rraw - bit rate on PHY layer [Mbps]

\begin{tabular}{|c|c|c|c|c|c|}
\hline & $802.11 b$ & $802.11 \mathrm{a}$ & 802.11g-only BSS & $\begin{array}{l}\text { 802.11g Protection } \\
\text { RTS/CTS }\end{array}$ & $802.11 n$ \\
\hline $\begin{array}{c}\mathbf{T}_{\text {Frame }} \\
{[\boldsymbol{\mu s}]}\end{array}$ & $444+\frac{8(l+78)}{R_{r a w}}$ & $94+\frac{8(l+64)+6}{R_{\text {raw }}}$ & $94+\frac{8(l+64)+6}{R_{\text {raw }}}$ & $520+\frac{8(l+234)+6}{R_{\text {raw }}}$ & $100+\frac{8(l+64)+6}{R_{\text {raw }}}$ \\
\hline
\end{tabular}


In Table 1 the delays in wireless links frames transfer are considered for 802.11 MAC layer specifications. Only DCF (Distributed Coordination Function) access method was under consideration. Implementation of DCF is mandatory for every WiFi device. On transport layer of the network UDP protocol is assumed and it carries application packets of small size (no packetization is performed as for Voice over IP, no fragmentation is supposed) and this is often a demand of automation control applications. The estimations give us minimal possible $T_{\text {Frame }}$ (no mechanism of contention window $\mathrm{CW}$ changes is taken into account; also no mechanism ARF (Auto Rate Fallback) is supposed).

In wireless channel no pass loss, fading and interference was supposed but those effects may be taken into account in analytical model by reducing maximal possible raw bit rate given by specifications of PHY layer to some lover bit rate (ARF mechanism). As one can conclude from figures in Table 1 frame transfer time is primarily determined by the structure of the frame and $R_{\text {raw }}$ is not so sufficient if packets are small.

Frame transfer time and bandwidth for 802.15.1

Analysing the characteristics of 802.15.1 or Bluetooth (BT) network that are important for the frame time transfer estimations we fallow to fundamental book (Tanenbaum and Wetherall, 2011). The architecture of BT is based on "piconet" structure:

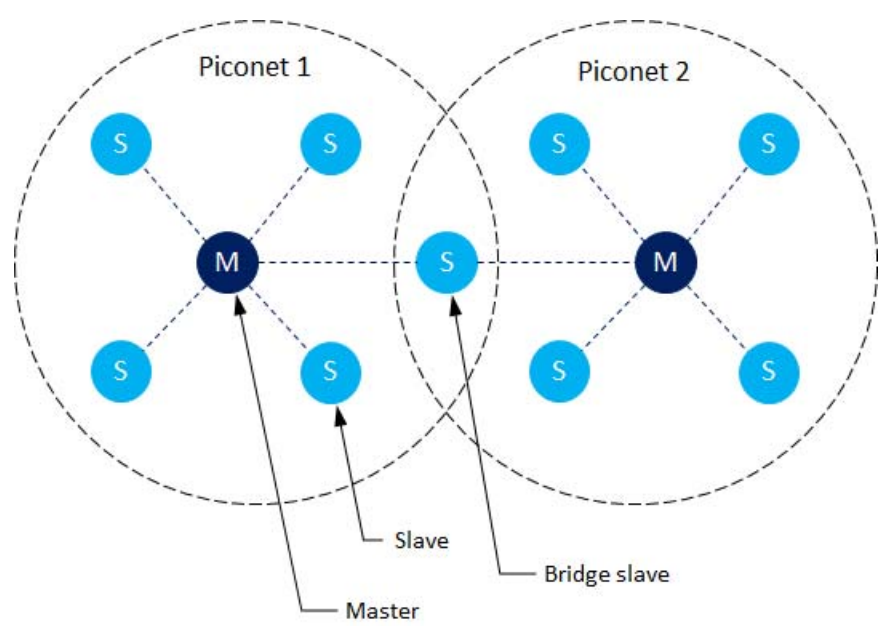

Figure 5. Architecture of Bluetooth network

In one piconet there is one Master and till 7 active Slave nodes. The data can be transferred only between Master and Slave, so data are transferred between Slaves only through Master. Due to the protocols stack two types of connections may be realized: SCO - Synchronous Connection Oriented and ACL Asynchronous Connectionless Link. For control application traffic transfer only ACL type is of interest.

On PHY level the shared radio resource is pseudo random sequence of channels (there are 79 frequency channels width of each is $1 \mathrm{MHz}$ ). TDM (Time Division Multiplexing) method is used for data transfer in piconet. As frequency hopping is used (FHSS - Frequency Hopping Spread Spectrum method) there is a slot time (frequency channel is changed every slot time). The slot time for 802.15 .1 is $625 \mu$ s or frequency is changed 1600 times per second. Master synchronizes the process for all nodes in piconet. For duplex data transfer in the connection between Master and Slave always pares exist - slots Master - Slave and slots Slave - Master. There are several bit rates (in our terms "raw") for data transfer on PHY level (Pahlavan and Krishnamurthy, 2009): 1 Mbps (modulation BFSK for Bluetooth 1.0); 2 Mbps (modulation PSK); 3 Mbps. On MAC (data link) layer the frames with appropriate structure exist. To transmit a frame 1,3 or 5 time slots may be used (Figure 6) and the structure of frame is different for that cases (Figure 7). 
a)

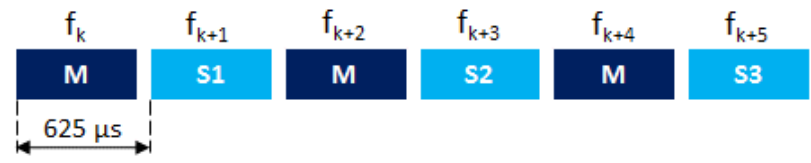

b)

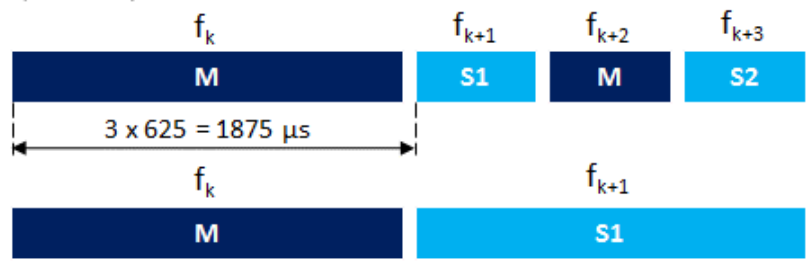

d)

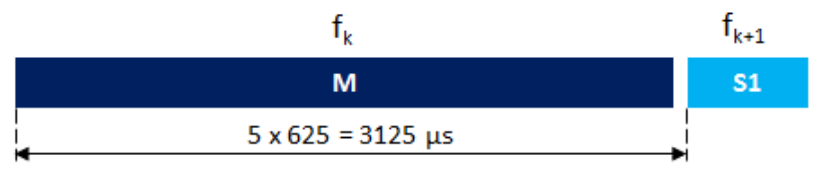

Figure 6. Transmission frames of different length: a) one-slot frame; b) asymmetric three-slot; c) symmetric three-slot; d) asymmetric five-slot

\begin{tabular}{|c|c|c|c|c|c|c|}
\hline \multicolumn{2}{|c|}{$\begin{array}{c}\text { Access code } \\
72 \text { bit }\end{array}$} & $\begin{array}{c}\text { Header } \\
54 \text { bit }\end{array}$ & \multicolumn{4}{|c|}{$\begin{array}{l}\text { Payload } \\
0-2744 \text { bit }\end{array}$} \\
\hline & & & $\begin{array}{l}\text { Data header } \\
8 \text { or } 16 \text { bit }\end{array}$ & \multicolumn{2}{|c|}{$\begin{array}{l}\text { Payload data } \\
0-2712 \text { bit }\end{array}$} & $\begin{array}{c}\text { CRC } \\
16 \text { bit }\end{array}$ \\
\hline DM1: & \multicolumn{2}{|c|}{ Header 8 bit } & Payload 0 & & $2 / 3 \mathrm{FEC}$ & \\
\hline DH1: & \multicolumn{2}{|c|}{ Header 8 bit } & \multicolumn{3}{|c|}{ Payload 0-216 bit } & \\
\hline DM3: & \multicolumn{2}{|c|}{ Header 16 bit } & Payload C & & $2 / 3 \mathrm{FEC}$ & \\
\hline DH3: & \multicolumn{2}{|c|}{ Header 16 bit } & \multicolumn{3}{|c|}{ Payload 0-1464 bit } & \\
\hline DM5: & \multicolumn{2}{|c|}{ Header 16 bit } & Payload 0 & bit & $2 / 3 \mathrm{FEC}$ & \\
\hline DH5: & \multicolumn{2}{|c|}{ Header 16 bit } & \multicolumn{3}{|c|}{ Payload 0-2712 bit } & \\
\hline
\end{tabular}

Figure 7. The frame structure for ACL (DM - Data Medium; DH - Data High; 1, 3, 5 - slots)

The maximum field size for data of packets coming on MAC level (payload on Figure 7) is defined by raw bit rate. On Figure 7 the payload length is shown for the raw bitrate 1Mbps. In (Tanenbaum and Wetherall, 2011) it is emphasized that only "payload" field is transmitted with speed 2 or 3 Mbps but the fields "Access code" and "Header" are always transmitted on speed 1Mbps. Table 2 demonstrates the ranges of "payload" length L for different frame structures ( $k$ - the number of slots for frame transfer) and raw speeds:

Table 2. Correspondence of payload length, $\mathrm{R}_{\mathrm{raw}}$ and number of slots for ACL frames without correction of errors (DH type)

\begin{tabular}{|l|l|l|l|}
\hline & $\mathbf{k}=\mathbf{1}$ & $\mathbf{k}=\mathbf{3}$ & $\mathbf{k = 5}$ \\
\hline $\mathbf{R}_{\text {raw }}=\mathbf{1}$ Mbps & $\mathrm{L}=0-27$ byte & $\mathrm{L}=28-183$ byte & $\mathrm{L}=184-339$ byte \\
\hline $\mathbf{R}_{\text {raw }}=\mathbf{2}$ Mbps & $\mathrm{L}=0-47$ byte & $\mathrm{L}=48-360$ byte & $\mathrm{L}=361-672$ byte \\
\hline $\mathbf{R}_{\text {raw }}=\mathbf{3}$ Mbps & $\mathrm{L}=0-76$ byte & $\mathrm{L}=77-545$ byte & $\mathrm{L}=546-1014$ byte \\
\hline
\end{tabular}


Thus, 802.15.1 technology is characterized by some mechanism of slot time reservation to transfer the stream of data packets using frames of different structure. This mechanism is in strong dependence on packets size. Moreover it depends on number of active nodes in piconet and possibilities of network adapters to work on higher speeds (for an example due to signal to noise ratio in radio channel).

Let us estimate the frame transfer time for the case when for packet streams in radio channel in piconet with one Master (it is always so) and $n$ Slave (according to the specifications $n \leq 7$ ) reservation mechanism gives time slot sequence as follows (see Figure 8):

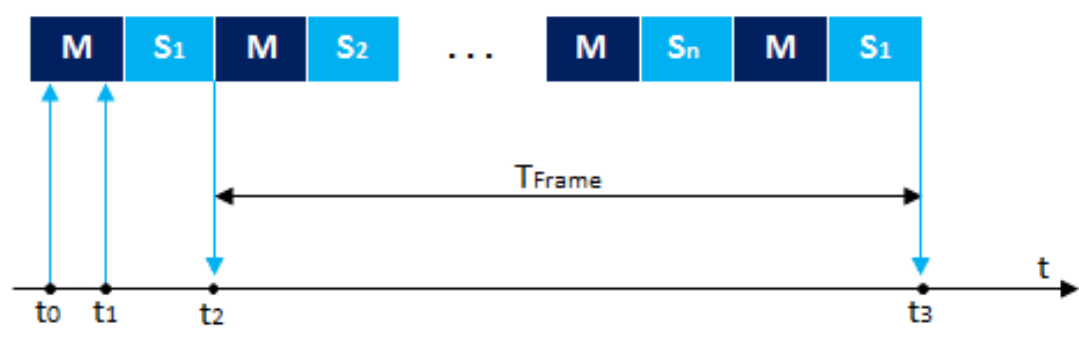

Figure 8. Time slot sequence for Master and n Slaves frames transfer. Here Master's and Slave's frames occupies the same number of slots

We have emphasize that for 802.15 .1 the one slot time is $625 \mu$ s and frame can occupy 1,3 or 5 time slots. It is obvious that:

$T_{\text {Frame }}=625 \cdot \sum_{i=1}^{n}\left(k_{M i}+k_{i}\right)=625 \cdot\left(k_{M} \cdot n+\sum_{i=1}^{n} k_{i}\right)[\mu s]$

Here $\mathrm{k}_{\mathrm{i}}$ - number of slots for frames from Slave $\mathrm{i}$ to Master, and $\mathrm{k}_{\mathrm{Mi}}$ - number of slots for frames from Master to Slave i. The equation (10) is an analog for $\mathrm{T}_{\text {Frame }}$ estimations for 802.15.1 as equations in Table 1 for 802.11 technologies.

It is not so easy to know what $\mathrm{k}$ will be in (10) due to lack of opacity for reservation mechanism. But we will suppose (and experiments confirm it) that reservation mechanism picks out minimal amount of slots for frames taking into account the packet size that comes on MAC layer. The ranges for packet sizes we have given in Table 2 .

For the demonstration haw our model of estimations works for 802.15.1 wireless data channel and "small" application packets ( $l=10$ byte, transport protocol UDP-IP with 28 additional bytes for MAC layer) we will consider two examples:

There are 2 nodes, - Master and Slave, so 2 streams of traffic are possible: Slave-Master and MasterSlave simultaneously; from Table 2 we have that it is possible to transmit such packets with frames of 1 slot on speed $2 \mathrm{Mbps}$ and the frame transfer time from (10) will be:

$T_{\text {Frame }}=625 \cdot\left(k_{M} \cdot n+\sum_{i=1}^{n} k_{i}\right)=625 \cdot(1 \cdot 1+1)=1250[\mu s]$

This estimation of $T_{\text {Frame }}$ is true for both directions. For the bandwidth B (maximal possible throughput) for both directions from (9) we receive:

$B=\frac{8 \cdot l}{T_{\text {Frame }}}=\frac{8 \cdot 10}{1250}=64[\mathrm{Kbps}]$

There are 3 nodes, - Master and two Slaves, so the traffics Slave1-Master, Slave2-Master are possible and in the revers directions: Master-Slave1, Master-Slave2; also traffics Slave1-Master-Slave2 and Slave2-Master-Slave1. The $T_{\text {Frame }}$ for one link from (10) will be:

$T_{\text {Frame }}=625 \cdot\left(k_{M} \cdot n+\sum_{i=1}^{n} k_{i}\right)=625 \cdot(1 \cdot 2+1+1)=2500[\mu s]$

For the traffics that not share the same link (for an example those traffics are Slave1-Master-Slave2 and Slave2-Master-Slave1) it is true:

$B=\frac{8 \cdot l}{T_{\text {Frame }}}=\frac{8 \cdot 10}{2500}=32[\mathrm{Kbps}]$ 


\section{Experiments on data transfer network}

To verify estimations that can be received from model described and frame transfer time calculations for 802.11 and 802.15.1 the set of experiments was performed. In experimental cases the architecture of network and structure of traffic were changed. In all cases the application payload was provided as a stream of "small" packets of size $l=12$ byte generated by utility Jperf. As a transport protocol UDP protocol was used. In all cases throughput and bandwidth (maximal throughput) were measured for data channels (paths) between Hosts. Wireless and wired links were composing channels.

\subsection{Experiments for 802.11}

In Table 3 the parameters of experiments are presented. As wireless technology $802.11 \mathrm{~g}$ specifications for wireless links were used. For column "Architecture" in Table 3 symbol "/" denotes wired link and symbol "_." denotes wireless link. In all experiments we have tried to maximize the ratios "signal to noise" (SNR) as far as possible, so it can be supposed that wireless adapters of Hosts worked on higher possible "raw" speed.

Table 3. Comparison of experimental and model results for WiFi experiments. Bandwidth for different network architectures and structures of traffic was compared

\begin{tabular}{|c|c|c|c|c|c|c|c|c|}
\hline \multicolumn{3}{|c|}{ Experiments } & \multirow{2}{*}{$\begin{array}{l}\text { Wireless } \\
\text { concurre } \\
\text { nt links } \\
\text { in } \\
\text { network }\end{array}$} & \multirow{2}{*}{$\begin{array}{l}\text { Wirele } \\
\text { ss } \\
\text { links } \\
\text { on } \\
\text { path }\end{array}$} & \multirow[b]{2}{*}{$\begin{array}{l}\text { Experimen } \\
\text { tal } \\
\text { bandwidth } \\
{[\mathrm{Kbps}]}\end{array}$} & \multirow[b]{2}{*}{$\begin{array}{l}\text { Model } \\
\text { bandwid } \\
\text { th } \\
{[\mathrm{Kbps}]}\end{array}$} & \multirow{2}{*}{$\begin{array}{l}\text { Deviation } \\
\text { Experime } \\
\text { nt from } \\
\text { Model } \\
\text { bandwidt } \\
\text { h [\%] }\end{array}$} & \multirow[b]{2}{*}{$\begin{array}{l}\text { Calculat } \\
\text { ed } \mathrm{T}_{\text {Frame }} \\
{[\mathrm{ms}]}\end{array}$} \\
\hline Name & Architecture & $\begin{array}{l}\text { Traffic } \\
\text { Bearer/BiDirecti } \\
\text { onal }\end{array}$ & & & & & & \\
\hline WiFi 1.1 & Host2-AP/Host1 & $\mathrm{B}$ & 1 & 1 & 848 & 911 & $-6,9$ & 0,105 \\
\hline WiFi 1.2 & Host2-AP/Host1 & $\mathrm{B}$ & 1 & 1 & 428 & 432 & $-0,9$ & 0,222 \\
\hline WiFi 2.1 & Host2-AP/Host1 & $\mathrm{BD}$ & 2 & 1 & 280 & 311 & $-10,0$ & 0,154 \\
\hline $\begin{array}{l}\mathrm{WiFi} \\
2.2 .1\end{array}$ & Host2-AP/Host1 & $\mathrm{BD}$ & 2 & 1 & 232 & 255 & $-9,0$ & 0,188 \\
\hline $\begin{array}{l}\mathrm{WiFi} \\
2.2 .2\end{array}$ & Host2-AP/Host1 & $\mathrm{BD}$ & 2 & 1 & 285 & 311 & $-8,4$ & 0,154 \\
\hline WiFi 3.1 & Host2-AP-Host1 & $\mathrm{B}$ & 2 & 2 & 252 & 255 & $-1,2$ & 0,188 \\
\hline WiFi 3.2 & Host2-AP-Host1 & $\mathrm{B}$ & 2 & 2 & 300 & 311 & $-3,5$ & 0,154 \\
\hline WiFi 4 & Host2-AP-Host1 & $\mathrm{BD}$ & 4 & 2 & 139 & 140 & $-0,7$ & 0,171 \\
\hline WiFi 5 & $\begin{array}{l}\text { Host1,Host2- } \\
\text { AP/Host3 }\end{array}$ & $\mathrm{B}$ & 2 & 1 & 249 & 255 & $-2,4$ & 0,188 \\
\hline WiFi 6 & $\begin{array}{l}\text { Host1,Host2-AP- } \\
\text { Host3 }\end{array}$ & $\mathrm{B}$ & 4 & 2 & 130 & 140 & $-7,1$ & 0,171 \\
\hline & & & & & & Average & $-5,0$ & 0,2 \\
\hline
\end{tabular}

The main conclusions from experiments are as follows. Our model is adequate to present the real process of packets transfer in the network with WiFi links; the predicted bandwidth of the channel is higher (approximately 5\%) than experimental one and this fact can be taking into account by more precisely the contention window (CW) changing mechanism. Frame transfer time (and application packet transfer time for "small packets") for the specification $802.11 \mathrm{~g}$ (and also for 802.11a,n) is about $0.2 \mathrm{~ms}$ per wireless link; this fact may be important for the real time applications packets streams.

\subsection{Experiments for 802.15.1}

In Table 4 the parameters of experiments are presented. 802.15.1 specifications for wireless links were used. In all experiments we have tried to maximize the ratios "signal to noise" (SNR) as far as possible, so it can be supposed that wireless adapters of Hosts worked on higher possible "raw" speed. 
Table 4. Comparison of experimental and model results for Bluetooth experiments. Bandwidth for different network architectures and structures of traffic was compared

\begin{tabular}{|c|c|c|c|c|c|c|c|c|}
\hline \multicolumn{3}{|c|}{ Experiments } & \multirow[b]{2}{*}{$\begin{array}{l}\text { Wireless } \\
\text { concurrent } \\
\text { links in } \\
\text { network }\end{array}$} & \multirow[b]{2}{*}{$\begin{array}{l}\text { Wireless } \\
\text { links on } \\
\text { path }\end{array}$} & \multirow[b]{2}{*}{$\begin{array}{l}\text { Experimental } \\
\text { bandwidth } \\
{[\mathrm{Kbps}]}\end{array}$} & \multirow[b]{2}{*}{$\begin{array}{l}\text { Model } \\
\text { bandwidth } \\
\text { [Kbps }]\end{array}$} & \multirow[b]{2}{*}{$\begin{array}{l}\text { Deviation } \\
\text { Experiment } \\
\text { from Model } \\
\text { bandwidth } \\
{[\%]}\end{array}$} & \multirow[b]{2}{*}{$\begin{array}{l}\text { Calculated } \\
\mathrm{T}_{\text {Frame }}[\mathrm{ms}]\end{array}$} \\
\hline Name & Architecture & $\begin{array}{l}\text { Traffic } \\
\text { Bearer/ } \\
\text { BiDirect } \\
\text { ional }\end{array}$ & & & & & & \\
\hline BT 1.1 & Master-Slave & $\mathrm{B}$ & 2 & 1 & 72 & 77 & $-6,6$ & 1,25 \\
\hline BT 1.2 & Slave-Master & B & 2 & 1 & 68 & 77 & $-12,0$ & 1,25 \\
\hline BT 2.1 & Master-Slave & BD & 2 & 1 & 70 & 77 & $-9,5$ & 1,25 \\
\hline BT 2.2 & Slave-Master & BD & 2 & 1 & 69 & 77 & $-10,5$ & 1,25 \\
\hline BT 3.1 & Master-Slave; Slave & B & 2 & 1 & 67 & 77 & $-12,4$ & 1,25 \\
\hline BT 3.2 & Slave-Master; Slave & B & 2 & 1 & 63 & 77 & $-18,4$ & 1,25 \\
\hline BT 4.1 & Master-Slave; Slave & $\mathrm{BD}$ & 2 & 1 & 65 & 77 & $-15,2$ & 1,25 \\
\hline BT 4.2 & Slave-Master; Slave & $\mathrm{BD}$ & 2 & 1 & 65 & 77 & $-14,8$ & 1,25 \\
\hline BT 5.1 & Slave1,Slave2-Master & B & 4 & 1 & 36 & 38 & $-5,5$ & 2,5 \\
\hline BT 5.2 & Slave1,Slave2-Master & B & 4 & 1 & 32 & 38 & $-16,4$ & 2,5 \\
\hline BT 6.1 & Slave1-Master-Slave2 & $\mathrm{B}$ & 4 & 2 & 34 & 38 & $-12,2$ & 2,5 \\
\hline BT 6.2 & Slave2-Master-Slave1 & B & 4 & 2 & 31 & 38 & $-18,2$ & 2,5 \\
\hline BT 7.1 & Slave1-Master-Slave2 & BD & 4 & 2 & 33 & 38 & $-14,3$ & 2,5 \\
\hline BT 7.2 & Slave2-Master-Slave1 & $\mathrm{BD}$ & 4 & 2 & 32 & 38 & $-16,4$ & 2,5 \\
\hline \multicolumn{7}{|l|}{ Average } & $-13,0$ & \\
\hline
\end{tabular}

The main conclusions from experiments are as follows. Our model is adequate to present the real process of packets transfer in the network with Bluetooth links; the predicted bandwidth of the channel is higher (approximately 13\%) than experimental one and this fact can be corrected taking into account more precisely the time slot reservation rescheduling mechanism. Smallest frame time (and application packet transfer time for "small packets") for 802.15 .1 is about $1.25 \mathrm{~ms}$ per wireless link (and is proportional to the number of active Slaves in piconet); this fact may be important for the real time applications packets streams.

\section{Conclusions}

In the matter of fact we have proposed analytical model for the estimations of application packets delay on their propagation paths through the network in what wireless links are present. It is done on the basis of Queueing Theory on network level and calculations of frames transfer time (Data Link or MAC layer) for wireless technologies 802.11 and 802.15.1. Analytical model gives the relationships between delays and bit rate on application level for different network architecture and traffic structures.

The delays for frames transfer are considered for 802.11 MAC layer specifications, only DCF (Distributed Coordination Function) access method was under consideration and PHY layer specifications $802.11 \mathrm{a} / \mathrm{b} / \mathrm{g} / \mathrm{n}$ were analyzed. For 802.15.1 specifications ACL (Asynchronous Connectionless Link) frame structure were taken into account. On transport layer of the network UDP protocol was used which carry application packets of small size (no packetization is performed) and this is often a demand of automation control applications. We have not considered here influence of pass loss, fading and interference in wireless channel.

The set of experiments were performed on WiFi and Bluetooth networks to validate the model and some of them are presented. It is shown that basic effects are taken into account and further accuracy "improvement" of the model will give not more than 5\%. As a result that is important for automation control applications we have reliably received the lowest possible level for packets delay, for 802.11 it is $0.2 \mathrm{~ms}$ and for 802.15.1 it is $1.25 \mathrm{~ms}$ (for one wireless link).

\section{Acknowledgements}

This paper has been published within the research project 'Research on dynamic wireless charging of electric vehicles and development of experimental model'. And it was carried out within grant program by European Regional Development Fund for general industrial research and for projects dealing with new 
product and technology developments. Latvian Investment and development agency Contract number: LKC-11-0002 project number: KC/2.1.2.1.1/10/01/008.

\section{References}

1. Krivchenkov, A. and Saltanovs, R. (2014) The performance analysis of WiFi data networks used in automation systems. In: Proceedings of the 14th International Conference "RELIABILITY and STATISTICS in TRANSPORTATION and COMMUNICATION” (RelStat'14), pp. 356-366.

2. Gast, M. (2002) 802.11 Wireless Networks: The Definitive Guide. O'Reilly, 464 pp.

3. Gast. M. (2003) When Is 54 Not Equal to 54? A Look at 802.11a, b, and g Throughput, published on Wireless DevCenter, http://www.oreillynet.com/wireless/.

4. Qiang Ni. (2005) Design and Analysis of MAC Protocol for IEEE 802.11n, published of Hamilton Institute, www.hamilton.ie/ncnrc/, $23 \mathrm{pp}$.

5. Tanenbaum, A.S. and Wetherall, D.J. (2011) Computer networks. 5th ed. Pearson Education, Inc., publishing as Prentice Hall.

6. Pahlavan, K. and Krishnamurthy, P. (2009) Networking Fundamentals: Wide, Local and Personal Area Communications, John Wiley \& Sons, Ltd., ISBN: 978-0-470-99289-0. 